\title{
¿DIFERENCIA LA VARIABLE GÉNERO A LA ÉLITE PARLAMENTARIA LATINOAMERICANA?
}

\author{
Araceli Mateos Díaz*
}

\section{INTRODUCCIÓN}

La participación de las mujeres en la vida e instituciones políticas está siendo cada vez más numerosa en los últimos años. Sin embargo, son muy pocas las mujeres que llegan a puestos de responsabilidad o de toma de decisiones

Las investigaciones que se han hecho desde las Ciencias Sociales sobre los cambios de las relaciones de las mujeres con la actividad política son escasas; y este número es aún menor en el caso de América Latina. Por ello, este artículo, dentro de su brevedad, y esperando poder realizar un análisis más completo de otros indicadores en un futuro, supone un acercamiento a la importancia que la variable género pueda tener, como elemento diferenciador, entre una serie de características de los miembros de las Cámaras de Diputados latinoamericanas.

Se pueden distinguir distintos niveles de élites políticas: aquellas que son elegidas como representantes en parlamentos, concejalías, etc; las que forman parte de grupos de interés y sindicatos y las que ocupan puestos en la administración. De estos tres niveles, los datos que se analizan en este artículo van a tener en cuenta el primero de ellos. Se trata de opiniones que miembros de Cámaras de Representantes de 18 países de América latina dieron a un amplio cuestionario perteneciente a la investigación "Élites Parlamentarias en América Latina” '. El análisis que aquí se hace se centra en cinco de esos 18 países: Argentina, Chile, Colombia, México y República Dominicana; pero también se tienen en cuenta las opiniones del conjunto de latinoamérica por género para poder comparar dentro de todo el conjunto de países investigados. La población está compuesta por un total de 969 entrevistados, de los cuales 844 son hombres y 125 mujeres.

En general, los estudios dirigidos a encontrar las diferencias entre hombres y mujeres se centran en analizar, por un lado, las características específicas del comportamiento de las mujeres: estudios sobre participación electoral ${ }^{2}$, autoposicionamiento ideológico en la escala izquierda-derecha ${ }^{3}$, estudios sobre el interés por la políti$\mathrm{ca}^{4} \mathrm{y}$, por otro lado, aquellos que analizan las características específicas de las mujeres políticas, o si el hecho de que sean mujeres las encargadas de diseñar unas determinadas políticas repercute en el tipo de ellas que sean implementadas 5 .

El objeto de estudio de este trabajo son las siguientes características específicas y personales de estos representantes: clase social de origen, posición socioeconómica, familiares dedicados a la política, dedicación exclusiva a la política y actividad antes de dedicarse a la política. El motivo por el que han sido escogidos estos países ha sido el mayor número de mujeres diputadas existentes en los mismos y que han sido entrevistadas, en comparación con el número de hombres que componen la Cámara ${ }^{6}$.

Resultados de estudios sobre élites políticas europeas muestran que, en términos generales, se pueden señalar unos rasgos básicos de estas élites políticas que son ampliables también a las mujeres que pertenecen a ellas: tienen orígenes de clase acomodados, con una escasa representación de la clase trabajadora, sobre todo en los partidos conservadores; su formación académica es elevada, origen urbano, con edad entre los 40-50 y, aunque la abogacía sigue siendo la profesión de origen principal del político profesional, existe un creciente número de otras profesiones como son la de profesor y periodista ${ }^{7}$. Pero no se subraya la imporMadrid

Becaria-Investigadora. Centro de Investigaciones Sociológicas.

1. Esta investigación ha sido dirigida por el profesor Manuel Alcántara de la Universidad de Salamanca y financiada por la Comisión Internacional de Ciencia y Tecnología (SEC95-0845).

2. Como estudios clásicos de participación política, cabe destacar el de Samuel Bernes y Max Kaase, 1979, "Political Action: Mass Participation in Five Western Democracies", Beverly Hills.

3. Para el caso español, véase el estudio de Pilar Brabo Castells, 1990, “Participación y Cultura política de las mujeres”, y el de Pilar PérezFuentes "Condicionamientos de la participación política de las mujeres", en Judith Astelarra, 1990, Participación política de las mujeres, Madrid, CIS, pp. 173-192.

4. "Mujeres y hombres de Europa hoy: actitudes ante Europa y la política", en Cuadernos de Mujeres de Europa, 1991, n. 35.

5. Edurne Uriarte: "Las mujeres en la élites políticas", en Mujeres en política, Edurne Uriarte y Arantxa Elizondo (coord.), Madrid, 1997.

6. A lo largo de este documento se utilizará el término "representante" para identificar a todos los miembros de la Cámara, sin distinción de género y, los términos "diputado" o "diputada" para los casos en que sí se quiera insistir en ello.

7. Edurne Uriarte: “Mujeres en las élites políticas”, en Mujeres en politica, Ariel, Madrid, 1997, pp. 53-75. 
tancia de estos rasgos como elementos diferenciadores entre hombres y mujeres. Con los datos obtenidos del objeto de estudio, anteriormente mencionado, lo que se trata de ver es si esos elementos que definen a las élites políticas europeas lo hacen también respecto a las latinoamericanas y, más concretamente, si se puede establecer alguna relación interesante teniendo en cuenta la variable sexo.

Las variables analizadas se dividen en tres grupos. El primer grupo: "elementos socioeconómicos" incluye las variables clase social de origen y posición socioeconómica subjetiva. El segundo grupo "tradición familiar": familiares dedicados a la política y número de familiares dedicados a la política. Y, finalmente, el tercer grupo incluye "elementos profesionales".

\section{ELEMENTOS SOCIOECONÓMICOS:}

La relación entre dedicarse a la política y pertenecer a clases sociales altas tiene antecedentes históricos, pero en el momento actual, y teniendo en cuenta la variable género, esta relación se hace aún más significativa para el conjunto de países latinoamericanos. Existe cerca de un 60 por ciento de las diputadas latinoamericanas que manifiesta que su clase social de origen es alta o media-alta, frente a ese mismo porcentaje de diputados que manifiesta que su clase social de origen es baja o media-baja (Cuadro I). mayor o menor porcentaje de miembros pertenecientes a una clase social u otra no es igual.

La segunda variable considerada, dentro de este grupo, es la posición socioeconómica. Esta variable es un indicador que proporciona información importante sobre el tipo de personas que componen la élite política y que puede diferenciarla del resto de sus conciudadanos. Sobre todo considerando las desigualdades sociales y económicas existentes en estos países.

La mayoría de los representantes latinoamericanos, un 64 por ciento, dice tener una posición socioeconómica acomodada. Un 31 por ciento del total califica su posición socioeconómica como modesta y tan sólo un 5 por ciento asegura que esta posición es rica. No hay prácticamente ningún representante que se considere muy rico (Cuadro II).

Teniendo en cuenta la variable género, no se presentan diferencias significativas en cuento a tener una posición socioeconómica rica o muy rica pero sí respecto a tener una posición acomodada o modesta. Así, existe un mayor porcentaje de diputados que de diputadas que asegura tener una posición modesta y, un mayor porcentaje de diputadas que de diputados que dice disfrutar de una posición acomodada.

El país donde la élite política se considera en una posición socioeconómica ventajosa respecto al resto de su país es Chile (sobre todo la élite femenina) ya que un 5,8 por ciento de los diputados y un 12,5 por ciento de sus diputa-

\section{Cuadro I: Clase social de origen ${ }^{8}$}

$\%$

\begin{tabular}{|c|c|c|c|c|c|c|c|c|c|c|c|c|}
\hline & \multicolumn{2}{|c|}{ Latinoamérica } & \multicolumn{2}{|c|}{ Argentina } & \multicolumn{2}{|c|}{ Colombia } & \multicolumn{2}{|c|}{ Chile } & \multicolumn{2}{|c|}{ México } & \multicolumn{2}{|c|}{ Rep. Dom. } \\
\hline & $\mathrm{H}$ & $\mathrm{M}$ & $\mathrm{H}$ & M & $\mathrm{H}$ & M & $\mathrm{H}$ & M & $\mathrm{H}$ & M & $\mathrm{H}$ & M \\
\hline Alta & 4,6 & 10,1 & - & 13,3 & 12,2 & 25,0 & 3,5 & - & 2,0 & 13,6 & - & 8,3 \\
\hline Media-alta & 35,0 & 48,1 & 35,9 & 33,3 & 44,9 & 50,0 & 58,8 & 87,5 & 24,2 & 50,0 & 38,0 & 41,7 \\
\hline Media-baja & 45,1 & 29,5 & 59,0 & 4,7 & 34,7 & 16,7 & 34,1 & 12,5 & 57,6 & 31,8 & 46,0 & 16,7 \\
\hline Baja & 15,2 & 12,4 & 5,1 & 6,7 & 8,2 & 2,3 & 3,5 & - & 16,2 & 4,5 & 16,0 & 33,3 \\
\hline $\mathrm{N}$ & 948 & 129 & 39 & 15 & 49 & 12 & 85 & 8 & 99 & 22 & 50 & 12 \\
\hline
\end{tabular}

De los países considerados, los representantes de Chile, Colombia y México son los que en un mayor porcentaje dicen pertenecer a una clase social de origen alta y mediaalta. Teniendo en cuenta el género, son también estos tres países en los que se encuentra un mayor porcentaje de diputados que dice pertenecer a una clase social de origen baja respecto al porcentaje de diputadas que así lo manifiesta. En Colombia existe el mayor porcentaje de diputadas que afirman que su clase social de origen es alta, un 25 por ciento; y en la República Dominicana el mayor porcentaje de ellas, un 33 por ciento, que considera esta clase social como baja.

Por tanto, puede decirse que el género es un elemento que establece diferencias entre las clases sociales de origen de los representantes latinoamericanos, pero al interior de cada país esta relación es diferente, la existencia de un das, dice tener una posición rica; y el casi 88 por ciento restante, en ambos sexos, tiene una posición acomodada. Seguidos de los representantes argentinos, donde siete de cada diez disfruta de una posición socioeconómica acomodada. En este último país no se observa ninguna diferencia por sexo .

Colombia, México y República Dominicana son los países donde la posición socioeconómica más común entre sus representantes es la modesta. Sorprende que si bien Colombia es el país donde un mayor número de sus representante aseguraba en el indicador anterior pertenecer a

8. La formulación de esta pregunta fue la siguiente: “¿A qué clase social de origen diría Ud. que pertenece su familia de origen? 1) Alta, 2) Media-alta, 3) Media-baja, 4) Baja. 


\section{Cuadro II: Posición socioeconómica ${ }^{9}$}

\begin{tabular}{l|rc|rc|rc|rc|cc|cc|}
\hline & \multicolumn{2}{|c|}{ Latinoamérica } & \multicolumn{2}{c|}{ Argentina } & \multicolumn{2}{c|}{ Colombia } & \multicolumn{2}{c|}{ Chile } & \multicolumn{2}{c|}{ México } & \multicolumn{2}{c}{ Rep. Dom. } \\
\hline & $\mathrm{H}$ & $\mathrm{M}$ & $\mathrm{H}$ & $\mathrm{M}$ & $\mathrm{H}$ & $\mathrm{M}$ & $\mathrm{H}$ & $\mathrm{M}$ & $\mathrm{H}$ & $\mathrm{M}$ & $\mathrm{H}$ & $\mathrm{M}$ \\
\hline Modesta & 35,9 & 26,6 & 27,9 & 26,7 & 49,0 & 41,7 & 3,5 & - & 45,5 & 21,7 & 50,0 & 41,7 \\
Acomodada & 59,0 & 68,5 & 72,1 & 73,3 & 49,0 & 58,3 & 89,5 & 87,5 & 48,5 & 73,9 & 48,0 & 58,3 \\
Rica & 5,0 & 4,8 & - & - & 2,0 & - & 5,8 & 12,5 & 6,1 & 4,3 & 2,0 & - \\
Muy rica & 0,1 & - & - & - & - & - & 1,2 & - & - & - & - & - \\
\hline $\mathrm{N}$ & 839 & 124 & 43 & 15 & 49 & 12 & 86 & 8 & 99 & 23 & 50 & 12 \\
\hline
\end{tabular}

una clase social de origen alta, no clasifiquen su posición socioeconómica como rica o muy rica sino únicamente como acomodada. Las diferencias por género más llamativas entre estos países se observan en México, donde las diputadas parecen poseer una mejor posición socioeconómica que los hombres. Un 25,4 por ciento más de mujeres que de hombres dice tener una posición acomodada. La República Dominicana es el país, de los considerados, que posee una élite política más "modesta" y Chile el país donde su élite política no incluye a personas de posición socioeconómica modesta.

Comparando estos datos con los resultados de los estudios referidos a las élites políticas europeas, puede decirse que en América Latina se cumple la premisa de que la mayoría (6 de cada 10 representantes) de la élite política tiene orígenes de clase acomodados. Lo que no coincide es la inexistencia de clase trabajadora entre sus miembros, ya que, como se ha visto, en torno al 30 por ciento de los representantes pertenecen a una clase social modesta.

\section{TRADICIÓN FAMILIAR}

Los mecanismos a través de los cuales se impide el acceso de las mujeres al poder político son muy diversos. Comenzando por la socialización y la transmisión de roles sociales tradicionales, según los cuales dentro de la pareja cada uno tiene un papel muy delimitado: el hombre es el encargado de desempeñar las tareas públicas, trabajo fuera de casa, etc, y la mujer las tareas del mundo privado: cuidado de los hijos, tareas del hogar, etc.; hasta llegar a elementos discriminatorios más sutiles, como justificar el lugar secundario que puede tener una mujer en una escala jerárquica, o el puesto que ocupa dentro de unas listas electorales, diciendo que las mujeres no tienen ambición, que no están lo suficientemente preparadas para un puesto específico o que sus responsabilidades como madre le restan tiempo a la hora de dedicarse a la política. En todo caso, la evidencia parece indicar que las mujeres que participan en política suelen ser tanto o más competentes que los hombres por lo que la oposición corresponde más a formas de discriminación que a selección en función de la capacidad ${ }^{10}$.
Pero lo que nunca se suele tener en cuenta es qué elementos, aunque sean pocos, y a excepción de las acciones positivas desarrolladas por los partidos o el gobierno, pueden favorecer la participación de las mujeres en política. Ser un personaje conocido de la vida social del país, ser esposa, hija o familiar de presidentes o de personas que se hayan dedicado o desempeñado algún tipo de actividad política importante es un aspecto que puede ayudar a la entrada, y el ascenso, de mujeres en la política.

Como se puede comprobar en el cuadro III, aunque para la totalidad de los países latinoamericanos el mismo porcentaje de diputados manifiesta haber tenido algún familiar que se haya dedicado a la política que los que aseguran lo contrario, en el caso de las diputadas, es mayor el porcentaje de ellas que sí han tenido familiares dedicados a la política (un 11,4 por ciento más).

Teniendo en cuenta el país al que pertenecen estos representantes, en Argentina, Colombia y México se comprueba la tónica general de que si bien dentro de los dos grupos hay un mayor porcentaje de ellos que ha tenido familiares que han desempeñado alguna actividad política, que los que no los han tenido, sigue siendo entre las diputadas donde este porcentaje es mayor.

Chile y las diputadas de la República Dominicana presentan un elemento común, la mitad de ellos/as han teniendo familiares en política, y la otra mitad no. El dato que más destaca de esta variable es que siete de cada diez diputados de la República Dominicana ha tenido familiares en política. México es el único país donde un mayor número de diputados no ha tenido familiares que hayan desempeñado alguna actividad relacionada con la política.

En muchos casos, los lazos familiares son decisivos para la llegada al poder de las mujeres en los respectivos países. Estos datos y la evidencia de las mayores dificultades de las mujeres para acceder al poder, sugieren la posibilidad de plantear la hipótesis de que la vinculación con familiares con influencia política es más importante

9. La formulación de la pregunta fue la siguiente: "Teniendo en cuenta las condiciones socioeconómicas de su paîs, ¿a qué clase social diría Ud. que pertenece? 1) Modesta, 2) Acomodada, 3 ) Rica, 4) Muy rica.

10. Judith Astelarra (comp.) Participación política de las mujeres, CIS, Madrid, 1987, pág. 18. 
Cuadro III: Familiares dedicados a la política ${ }^{11}$

$\%$

\begin{tabular}{l|cc|cc|cc|cc|cc|cc|}
\hline & \multicolumn{2}{|c|}{ Latinoamérica } & \multicolumn{2}{|c|}{ Argentina } & \multicolumn{2}{c|}{ Colombia } & \multicolumn{2}{c|}{ Chile } & \multicolumn{2}{c|}{ México } & \multicolumn{2}{c}{ Rep. Dom. } \\
\hline & H & M & H & M & H & M & H & M & H & M & H & M \\
\hline Sí & 50,3 & 55,7 & 57,1 & 60,0 & 62,7 & 66,7 & 50,0 & 50,0 & 41,4 & 56,5 & 70,0 & 50,0 \\
No & 49,7 & 44,3 & 42,9 & 40,0 & 37,3 & 33,3 & 50,0 & 50,0 & 58,6 & 43,5 & 30,0 & 50,0 \\
\hline N & 953 & 131 & 42 & 15 & 51 & 12 & 84 & 8 & 99 & 23 & 50 & 12 \\
\hline
\end{tabular}

en las mujeres que en los hombres en su carrera hacia el poder ${ }^{12}$

Por lo tanto, en términos generales, puede decirse que una característica de la élite política latinoamericana es tener o haber tenido familiares dedicados a la política. Y que esta cantidad es bastante alta. En torno al 50 por ciento de diputados y diputadas ha tenido cuatro o más miembros de su familia desarrollando actividades parecidas a las suyas en la política.

Los representantes de Argentina y República Dominicana son los que poseen más número de familiares en política, seguidos de los colombianos. Los chilenos y los mexicanos destacan por tener, mayoritariamente, un solo familiar que ha desarrollado actividades políticas. De todos estos países, las diputadas de República Dominicana y las de Argentina son las que cuentan con un mayor número de familiares políticos (cuatro o más) que los hombres, un 20,1 por ciento y un 10,9 por ciento, respectivamente. Las diferencias por sexo de nuevo vienen influidas por el país considerado. sabilizándose de la mayor parte de las tareas del hogar y del cuidado de los hijos. La doble o triple jornada laboral que tiene algunas mujeres les hace imposible su dedicación a la política.

Dado que para ser nombrada candidata no sólo hay que demostrar determinadas cualidades, sino, además, invertir una gran cantidad de tiempo en actividades de partido, las mujeres se enfrentan a especiales dificultades para encontrar tiempo para esa actividad extra ${ }^{13}$. En algunos países incluso, la legislación electoral prohibe compatibilizar la actividad parlamentaria con otras tareas o con su profesión anterior antes de ser elegido como representante.

La mitad de los diputados latinoamericanos compatibiliza la política con otras tareas, y tres de cada cuatro diputadas también. En todos los países considerados en el análisis, salvo en la República Dominicana, donde ocho de cada diez diputadas asegura dedicarse exclusivamente a la política, la mayoría de sus representantes se dedica a otras tareas, además de la parlamentaria. Siendo Chile y Colombia, los países donde las mujeres compatibilizan más

\section{Cuadro IV: Número de familiares dedicados a la política}

$\%$

\begin{tabular}{|c|c|c|c|c|c|c|c|c|c|c|c|c|}
\hline & \multicolumn{2}{|c|}{ Latinoamérica } & \multicolumn{2}{|c|}{ Argentina } & \multicolumn{2}{|c|}{ Colombia } & \multicolumn{2}{|c|}{ Chile } & \multicolumn{2}{|c|}{ México } & \multicolumn{2}{|c|}{ Rep. Dom. } \\
\hline & $\mathrm{H}$ & M & $\mathrm{H}$ & M & $\mathrm{H}$ & M & $\mathrm{H}$ & M & $\mathrm{H}$ & M & $\mathrm{H}$ & M \\
\hline Uno & 25,4 & 24,4 & 14,0 & 20,0 & 29,0 & 62,5 & 66,7 & 50,0 & 56,1 & 53,8 & 16,0 & 8,3 \\
\hline Dos & 9,8 & 11,8 & 23,3 & - & 22,6 & - & 9,5 & 50,0 & 29,3 & 15,4 & 18,0 & 16,7 \\
\hline Tres & 8,9 & 11,8 & 7,0 & 13,3 & 16,1 & - & 23,8 & - & 12,2 & 30,8 & 12,0 & - \\
\hline Cuatro o más & 55,8 & 52,0 & 55,8 & 66,7 & 32,3 & 37,5 & - & - & 2,4 & - & 54,0 & 75,0 \\
\hline $\mathrm{N}$ & 908 & 127 & 53 & 15 & 31 & 8 & 4 & 4 & 41 & 13 & 50 & 12 \\
\hline
\end{tabular}

\section{ELEMENTOS PROFESIONALES}

El desempeño de actividades políticas exige una gran dedicación de tiempo; y este ha sido uno de los argumentos utilizados para justificar la no participación de las mujeres en política. La cuestión del tiempo disponible de las mujeres ha estado condicionada a lo que se consideraba como responsabilidades exclusivas de la mujer, las responsabilidades familiares. Pero es que incluso las mujeres que desempeñan una profesión fuera del hogar, siguen respon- esta actividad: el 87,5 y el 91,7 por ciento, respectivamente (cuadro V).

No tiene ninguna relación el pertenecer a clases sociales altas y dedicarse exclusivamente a la política, ya que las diputadas pertenecen a clase social alta y sin embargo, tres

11. La pregunta fue formulada de la siguiente manera: ¿Tiene Ud. familiares que se hayan dedicado a la política? 1) Sí, 2) No.

12. Uriarte, E., op. cit., pág. 63.

13. Uriarte, E., op. cit., pág. 67. 


\section{Cuadro V: Dedicación exclusiva a la política}

$\%$

\begin{tabular}{l|cc|cc|cc|cc|cc|cc|}
\hline & \multicolumn{2}{|c|}{ Latinoamérica } & \multicolumn{2}{|c|}{ Argentina } & \multicolumn{2}{|c|}{ Colombia } & \multicolumn{2}{c|}{ Chile } & \multicolumn{2}{c|}{ México } & \multicolumn{2}{c}{ Rep. Dom. } \\
\hline & H & M & H & M & H & M & H & M & H & M & H & M \\
\hline Sí & 48,5 & 24,0 & 28,6 & 33,3 & 52,9 & 8,3 & 23,3 & 12,5 & 37,4 & 17,4 & 80,0 & 58,3 \\
No & 51,5 & 76,0 & 71,4 & 66,7 & 47,1 & 91,7 & 76,7 & 87,5 & 62,6 & 82,6 & 20,0 & 41,7 \\
\hline N & 953 & 129 & 42 & 15 & 51 & 12 & 86 & 8 & 99 & 23 & 50 & 12 \\
\hline
\end{tabular}

de cada cuatro de ellas asegura no dedicarse solamente a la política.

Dentro de la gran cantidad de aspectos que son utilizados para justificar la escasa participación y presencia de mujeres en política se encuentra su falta de preparación profesional y política. Hace unas décadas éste podía ser un argumento válido para marcar diferencias entre hombres y mujeres puesto que el acceso a la enseñanza superior estaba muy limitado para ellas. En cambio, actualmente, la formación de las mujeres es semejante o superior a la de los hombres. Lo que sí existe es una mayor presencia de hombres en carreras técnicas superiores y de mujeres en las carreras jurídicas y sociales ${ }^{14}$. diputados (el 28,6 por ciento) como diputadas $(23,6$ por ciento) son profesiones liberales como la medicina, arquitectura, periodismo, etc.

La segunda área de actividad más común entre ellos está relacionada con la justicia: abogados, notarios, jueces, etc. El 18,9 por ciento y el 18,2 de diputados y diputadas, respectivamente, afirman tener y haber desempeñado esas profesiones. El que la abogacía sea una de las profesiones más comunes entre los representantes latinoamericanos coincide con los resultados de otros estudios de élites políticas en países europeos ${ }^{15}$.

El tercer grupo de profesiones que incluye a un mayor número de ellos comienza a establecer diferencias

\section{Cuadro VI: Actividad antes de dedicarse a la política}

\begin{tabular}{|c|c|c|c|c|c|c|c|c|c|c|c|c|}
\hline & \multicolumn{2}{|c|}{ Latinoamérica } & \multicolumn{2}{|c|}{ Argentina } & \multicolumn{2}{|c|}{ Colombia } & \multicolumn{2}{|c|}{ Chile } & \multicolumn{2}{|c|}{ México } & \multicolumn{2}{|c|}{ Rep. Dom. } \\
\hline & $\mathrm{H}$ & M & $\mathrm{H}$ & M & $\mathrm{H}$ & M & $\mathrm{H}$ & M & $\mathrm{H}$ & $M$ & $\mathrm{H}$ & $M$ \\
\hline Político, sindicatos & 2,3 & 5,5 & - & 6,7 & - & 8,3 & 2,3 & - & 5,2 & 17,4 & - & - \\
\hline Abogados, jueces & 18,9 & 18,2 & 17,5 & 20,0 & 22,0 & 41,7 & 38,4 & 12,5 & 15,6 & 4,3 & 16,0 & 25,0 \\
\hline $\begin{array}{l}\text { Otras profesiones } \\
\text { liberales }\end{array}$ & 28,6 & 23,6 & 35,0 & 20,0 & 24,0 & 8,3 & 31,4 & 50,0 & 22,9 & 21,7 & 8,0 & 16,7 \\
\hline $\begin{array}{l}\text { Empleados sector } \\
\text { público }\end{array}$ & 5,8 & 9,1 & - & 13,4 & 2,0 & - & 3,5 & - & 9,3 & 17,3 & 6,0 & 8,3 \\
\hline $\begin{array}{l}\text { Grandes empresarios } \\
\text { y directivos } \\
\text { de empresas }\end{array}$ & 7,6 & 2,7 & 15,0 & - & 12,0 & - & 5,8 & - & 5,2 & - & 4,0 & - \\
\hline $\begin{array}{l}\text { Pequeños } \\
\text { empresarios }\end{array}$ & 9,5 & 6,3 & 10,0 & - & 8,0 & 16,7 & 5,9 & - & 4,1 & 4,3 & 28,0 & - \\
\hline $\begin{array}{l}\text { Profesores } \\
\text { universitarios }\end{array}$ & 7,6 & 10,0 & - & 6,7 & 6,0 & 16,7 & 5,8 & 12,5 & 12,5 & 8,7 & 2,0 & - \\
\hline Otros profesores & 6,8 & 16,4 & 7,5 & 20,0 & 2,0 & 8,3 & 2,3 & 25,0 & 9,4 & 22,7 & 6,0 & 8,3 \\
\hline $\begin{array}{l}\text { Trabajadores agrícolas } \\
\text { e industriales }\end{array}$ & 3,0 & - & - & - & 6,0 & - & - & - & 6,3 & - & 2,0 & - \\
\hline $\begin{array}{l}\text { Trabajadores sector } \\
\text { ervicios }\end{array}$ & 2 & 0,9 & 5,0 & 6,7 & 4,0 & - & 1,2 & - & 3,1 & - & 16,0 & - \\
\hline Militares & 0,8 & - & 5,0 & - & - & - & - & - & 1,0 & - & 2,0 & - \\
\hline Otras & 6,4 & 7,3 & 5,0 & 6,7 & 14,0 & - & 4,6 & - & 5,2 & 4,3 & 10,0 & 41,7 \\
\hline
\end{tabular}

En el caso de las profesiones o actividades desarrolladas antes de dedicarse a la política o que siguen desempeñando, se pueden distinguir ciertas tendencias (ver cuadro VI). La principal actividad a la que se dedicaban tanto
14. Las españolas en el umbral del $S$. XXI, Instituto de la Mujer, Madrid, 1994, págs. 132-133.

15. Interparliamentary union, participaction of women in political life and in the dicesion-making process, Ginebra, 1988. 
por sexo. La tercera rama de actividad más común entre los diputados es la empresarial, el 95 por ciento de ellos son pequeños empresarios y el 7,6 por ciento grandes empresarios y directivos de empresas. En cambio, entre las diputadas, el 16,4 por ciento son profesoras de niveles medios y el 10 por ciento profesoras universitarias. No hay ninguna diputada que trabaje en actividades agrícolas o industriales.

La principal diferencia que se observa en los países analizados es que entre las diputadas no hay ninguna que sea gran empresaria o directiva; y entre los diputados prácticamente ninguno antes de dedicarse a la política tenía una actividad relacionada con ella (pertenecía a alguna otra organización política o sindical), la presencia de militares en la élite política latinoamericana es casi inexistente.

\section{LAS PRINCIPALES DIFERENCIAS}

De todo lo presentado anteriormente pueden establecerse como características de las diputadas latinoamericanas las siguiente: pertenecen a la clase media-alta, gozan de una posición socioeconómica acomodada, han tenido familiares dedicados a la política, dos de cada cuatro diputadas ha tenido en su familia cuatro miembros o más dedicados o relacionados con la política. Las tres cuartas partes de ellas no se dedican exclusivamente a la tarea legislativa sino que compaginan ésta con su profesión anterior. Y entre las profesiones más comunes de las diputadas se encuentran la medicina, arquitectura y la enseñanza.

Los diputados se consideran, mayoritariamente, de clase social de origen baja, con una posición socioeconómica acomodada. La mitad de ellos ha tenido o tiene familiares dedicados a la política y, la cifra más común de familiares dedicados a la política, asciende a cuatro o más. Su dedicación a esta actividad legislativa no es exclusiva, la mitad la compagina con su profesión. Y las profesiones más comunes entre estos diputados están las profesiones liberales y la actividad empresarial.

Pero estas características no marcan una gran diferencia entre ambos grupos con lo que no puede concluirse que el género, considerando todos los países en conjunto, diferencie a diputados y diputadas, aunque en algún momento sí lo haga en algunas de las características y considerando los países por separado.

Lo que sí puede concluirse es que algunos de los razonamientos que viene dándose para explicar la escasa presencia de mujeres en la política no se cumplen según los datos aquí presentados.

\section{BIBLIOGRAFÍA}

AstelarRA, Judith (1990): Participación politica de las mujeres, Madrid, CIS.

Barnes, Samuel y Kasse, Max (1979): Political Action: Mass Participation in Five Western Democracies, Beverly Hills, Sage.

Bravo, Pilar (1990): "Participación y cultura política de las mujeres”, en Judith Astelarra: Participación politica de las mujeres, Madrid, CIS.

Cuadernos de Mujeres de Europa (1991), n 35: "Mujeres y hombres de Europa hoy: actitudes ante Europa y la política".

Interparliamentary union, participation of women in political life and in the dicesion-making process, Ginebra, 1988.

Las españolas ante el umbral del S.XXI, Instiuto de la Mujer, Madrid, 1994.

La mujer latinoamericana ante el reto del S.XXI. IX Jornadas de investigacióninterdisciplinaria sobre la mujer. UAM, Madrid, 1996.

URIARTE, Edurne y ELIZONDO, Arantxa (1997): Mujeres en política, Madrid, Ariel.

\section{RESUMEN}

Este artículo pretende definir la relevancia de la variable género como elemento diferenciador dentro de los representantes parlamentarios de una muestra de cinco países (Argentina, Chile, Colombia, México y República Dominicana). Para ello, se tienen en cuenta una serie de variables: clase social de origen, posición socioeconómica, familiares dedicados a la política, dedicación exclusiva a la política y la actividad del diputado antes de dedicarse a la política. El análisis pormenorizado de estas características no permite considerar al género como elemento que diferencie a diputados y diputadas para el conjunto de países analizados. Y es que las conclusiones varían si analizamos los casos mencionados por separado.

\section{ABSTRACT}

This article analyses the implications of gender among the paliamentarians of Argentina, Chile, Colombia, Mexico and the Dominican Republic. With that purpose, it analyses the parliamentarians' class origins, their socioeconomic position, the political involvement of their relatives and the activies of deputies before becoming professional politicians. This analysis leads to the conclusion that gender is not a common source of variation among the parliamentarians of all these five countries. The reason for this is that the role of gender seems to vary from country to country. 